\title{
Effects of one- and three-day binge alcohol exposure in neonatal C57BL/6 mice on spatial learning and memory in adolescence and adulthood
}

\author{
Jennifer L. Wagner ${ }^{\mathrm{a},{ }^{*}}$, Feng C. Zhou ${ }^{\mathrm{b}}$, and Charles R. Goodlett ${ }^{\mathrm{a}}$ \\ aDepartment of Psychology, Indiana University-Purdue University Indianapolis, Indianapolis, IN \\ 46202, USA \\ bepartment of Anatomy and Cell Biology, Indiana University School of Medicine, Indianapolis, IN \\ 46202, USA
}

\section{Abstract}

Binge-like alcohol exposure during the early postnatal period in rats and mice causes deficits in spatial learning and memory that persist into adulthood. Wozniak et al. (2004) reported that heavy binge alcohol exposure on postnatal day 7 (PD 7) in C57BL/6 (B6) mice produced profound spatial learning deficits in the Morris water maze when tested in adolescence (P30-39); when tested in adulthood, however, the deficits were greatly attenuated. Using a similar PD 7 binge alcohol exposure paradigm in B6 mice, we tested whether a single-day (PD 7 only) alcohol treatment produced place learning deficits in both adolescence and in adulthood, and further tested whether a more extended (3-day, PD 7-9) alcohol exposure would induce more severe and enduring deficits. B6 mice were given either 2 subcutaneous injections of alcohol $(2.5 \mathrm{~g} / \mathrm{kg}$ each) $2 \mathrm{~h}$ apart on PD 7 or on PD 7-9, and compared with controls that received saline vehicle injections and controls that received no injections. The alcohol injections on PD 7 produced average peak blood alcohol concentrations of $472 \mathrm{mg} / \mathrm{dL}$ and evoked typical patterns of activated caspase-3positive neurons in the cortex, hippocampal formation, and striatum $6 \mathrm{~h}$ after the last injection. Mice were given standard place training or random location training in the Morris water maze either as adolescents (PD 30-39) or adults (PD 70-79). The adolescents acquired the place learning more slowly than adults, and the alcohol treatments produced only modest place acquisition deficits. In contrast, both the PD7 and the PD 7-9 alcohol treatments resulted in large and significant spatial learning impairments in adults. In contrast to the previous findings of Wozniak et al. (2004), these results indicate that binge alcohol exposure in the 3rd trimester equivalent produces significant and enduring deficits in spatial learning in B6 mice.

\section{Keywords}

Morris water maze; Fetal alcohol spectrum disorders; Neurobehavioral deficits; Neurodevelopmental deficits

\footnotetext{
(C) 2014 Elsevier Inc. All rights reserved.

*Corresponding author. Present address: Department of Pharmaceutical Sciences, College of Pharmacy, University of Kentucky, 789 S. Limestone St., BPC 498, Lexington, KY 40536, USA. Tel.: +1 859323 5783; fax: +1 859257 7585. jennifer.wagner@uky.edu (J.L. Wagner).
} 


\section{Introduction}

Prenatal alcohol exposure is known to produce varying degrees of lasting craniofacial, neuroanatomical, and neurobehavioral abnormalities, collectively known as fetal alcohol spectrum disorder (FASD); for review see Kodituwakku (2007) and Riley and McGee (2005). Rodent models of FASD have revealed multiple sensitive periods over early development during which alcohol exposure can have severe effects on specific developmental processes in the embryo or fetus (Cudd, 2005; Dobbing \& Sands, 1979; Dobbing \& Smart, 1974; Rice \& Barone, 2000). Numerous studies have identified the rodent "neonatal brain growth spurt," sometimes termed the 3rd trimester equivalent, to be a period of vulnerability to alcohol-induced neuronal loss (Cudd, 2005; Dobbing \& Sands, 1979). Heavy binge-like alcohol exposure during this early postnatal period in rodents has been shown to produce dose-dependent apoptosis and enduring reductions in neuronal populations in several brain regions including the cerebral cortex, hippocampal formation, anterior thalamus, mammillary bodies, and cerebellum (Ikonomidou et al., 2000; Light, Belcher, \& Pierce, 2002; Livy, Miller, Maier, \& West, 2003; Tran \& Kelly, 2003; West \& Goodlett, 1990; Wozniak et al., 2004). Binge alcohol exposure during this period also has been shown to result in a number of neurobehavioral deficits, including deficits in spatial learning and memory that can persist into adulthood in rats (Clements, Girard, Ellard, \& Wainwright, 2005; Girard, Xing, Ward, \& Wainwright, 2000; Goodlett, Kelly, \& West, 1987; Johnson \& Goodlett, 2002; Kelly, Goodlett, Hulsether, \& West, 1988; O’LearyMoore, McMechan, Mathison, Berman, \& Hannigan, 2006; Pauli, Wilce, \& Bedi, 1995; Tomlinson, Wilce, \& Bedi, 1998) and in C57BL/6 inbred (Wozniak et al., 2004) and ddYoutbred albino mice (Furumiya \& Hashimoto, 2011).

Olney and colleagues have extensively studied a rodent model of a single episode of binge alcohol exposure during the 3rd trimester equivalent using 2 subcutaneous injections of 2.5 $\mathrm{g} / \mathrm{kg}$ alcohol, given $2 \mathrm{~h}$ apart, producing peak blood alcohol concentrations (BACs) greater than $500 \mathrm{mg} / \mathrm{dL}$ and that remained above $200 \mathrm{mg} / \mathrm{dL}$ for more than $6 \mathrm{~h}$ after the second injection (Ikonomidou et al., 2000, 1999; Wozniak et al., 2004). This model of extreme binge exposure has been shown to cause a wave of apoptotic neurodegeneration, including activation of caspase-3, the appearance of TUNEL-positive cells, and positive DeOlmos silver staining in the cerebral cortex, hippocampal formation, retrosplenial cortex, mammillary bodies, anterior thalamic nuclei, and basal ganglia (Coleman, Oguz, Lee, Styner, \& Crews, 2012; Ikonomidou et al., 2000, 1999; Olney et al., 2002; Wozniak et al., 2004), with the largest effects elicited by treatment on postnatal day (PD) 7.

Using this model of heavy binge alcohol exposure on PD 7 in C57BL/6 mice, Wozniak et al. (2004) showed profound spatial learning deficits in the Morris water maze task when the mice were tested in adolescence (PD 30-39). However, when other groups of mice were tested in adulthood (10 weeks old), spatial learning deficits were evident but were greatly attenuated compared to the adolescent groups. The authors interpreted this as evidence of recovery of function potentially attributable to adult neurogenesis. In contrast, Furumiya and Hashimoto (2011) recently reported that outbred (albino) ddY mice given the same bingelike alcohol exposure used by Wozniak et al. (2004) showed significant and large place 
learning deficits when tested at 7 weeks of age, and the effects persisted even when re-tested at 12 weeks. Procedural differences between the two reports make it difficult to resolve the markedly different outcomes in the adult mice reported in these two studies.

The profound deficits in adolescent mice reported by Wozniak et al. (2004) could be due to either the significant neonatal alcohol-induced loss of neurons in the forebrain networks involved in spatial learning, to major delays in the development of these networks, or both. If plasticity and reorganization of neural networks during and after adolescence can resolve profound spatial learning deficits, this would have important implications for the expression and treatment of cognitive deficits in children with FASD. In contrast, if major deficits in spatial learning persist into full adulthood, as indicated by Furumiya and Hashimoto (2011) and by previous studies in rat models of 3rd trimester binge alcohol exposure (Johnson \& Goodlett, 2002; Pauli et al., 1995; Tomlinson et al., 1998), recovery of function of spatial information processing would be an unexpected trajectory of neurobehavioral development from adolescence into adulthood.

The main purpose of the current study was to compare neonatal alcohol-induced spatial learning performance in the Morris water maze in adolescence and adulthood using the same alcohol administration protocol on PD 7 employed by Wozniak et al. (2004). The current study also sought to extend these findings to include a 3-day binge exposure group, which was expected to produce more severe deficits that would last well into adulthood. Further, the current study assessed spatial learning for each neonatal treatment condition by comparing groups given typical spatial navigation training (same location across all trials) to separate performance control groups given random training (different random locations across all trials). With these training procedures (Morris, 1981; Morris, Garrud, Rawlins, \& O'Keefe, 1982), the spatial learning of the Place-trained groups can be clearly demonstrated by the emergence of significantly shorter path lengths and escape latencies, relative to the Random group (trained with an unpredictable platform location). We tested two main hypotheses derived from the previous findings of Wozniak et al. (2004): 1) in adolescents, spatial learning will be evident from the differences between Place- and Random-trained groups, and both alcohol treatments would produce large spatial learning deficits, and 2) adults would show more efficient spatial learning than adolescents, regardless of neonatal treatment, and the spatial learning deficits of the PD 7 alcohol-treated group would be attenuated, whereas the PD 7-9 alcohol-treated group would show large spatial learning deficits.

\section{Materials and methods}

\section{Subjects}

Time-mated pregnant C57BL/6 dams were purchased from Harlan (Indianapolis, IN) and housed in the Indiana University-Purdue University Indianapolis School of Science vivarium. The vivarium was maintained on a 12-h/12-h light/dark cycle, lights on at 7:00 AM. Beginning on gestational day 17, identified from the reported sperm-positive date, mice were inspected daily at 5:00 PM. Litters (or partial litters) found at that time on a given day were designated as postnatal day 0 (P0). On PD 1, litters were culled to 6 pups ( 3 males and 3 females when possible). Pups from 48 litters were randomly assigned within litter to 1 of 3 
injection treatment groups: alcohol on PD 7 (and saline on PD 8-9); alcohol on PD 7-9; or saline on PD 7-9. Eighteen other litters were assigned to suckle-control conditions and were weighed on the same schedule as treated litters. During injection treatments, pups were identified by marking on the ventrum and paws with permanent black marker, reapplied before each injection to ensure legibility. On PD 10, pups received subcutaneous injections of a small volume of sterile India ink under one or more paws to provide a permanent, unique identifier for each littermate. Upon weaning (PD 21), mice were group-housed by sex with ad libitum access to food and water. Body weights were obtained daily for all pups from PD 7 through PD 12, then again on PD 15, 21, and 25. All protocols were in accordance with NIH guidelines and approved in advance by the IUPUI Institutional Animal Care and Use Committee.

\section{Alcohol treatment}

On PD 7, male and female pups of the litters assigned to injection treatments were randomly assigned by sex to 1 of 3 treatment groups (alcohol on PD 7 and saline on PD 8-9; alcohol daily on PD 7-9; saline control on PD 7-9). The PD 7 alcohol treatment was similar to that of Wozniak et al. (2004). Alcohol was given in 2 daily subcutaneous injections ( $2 \mathrm{~h}$ apart) in a dose of $2.5 \mathrm{~g} / \mathrm{kg}$ body weight (per injection) in a concentration of $15 \% \mathrm{w} / \mathrm{v}$ ethanol in $0.9 \%$ $(\mathrm{w} / \mathrm{v})$ sterile saline, in a volume of $16.67 \mathrm{~mL} / \mathrm{kg}$ (total daily dose of $5.0 \mathrm{~g} / \mathrm{kg}$ ). The PD 7 alcohol group was injected with alcohol on PD 7 and saline on PD 8-9. Mice in the PD 7-9 alcohol group were given the 2 daily alcohol treatments for all 3 days. Saline-control injections were given subcutaneously parallel to the alcohol groups. Injections were given between 0800 and $1200 \mathrm{~h}$ on PD 7-9. During the injection procedure, pups were removed from the dam as a litter, and placed in a huddle on a $37^{\circ} \mathrm{C}$ heating pad. Each round of injections took no more than $10 \mathrm{~min}$, and the pups were immediately placed back (as a litter) with the dam and returned to the vivarium until the next round of injections.

Offspring from 18 other litters served as suckle controls and were handled and weighed during the same schedule as treated offspring.

\section{Blood alcohol concentrations (BACs)}

Trunk blood samples were collected in heparinized centrifuge tubes throughout the experiment from separate litters of mice (10 litters, $n=36$ ), 1, 4 or $7 \mathrm{~h}$ after the last alcohol injection on PD 7. BACs were assayed from the plasma of each sample using an Analox ${ }^{\circledR}$ GL5 Alcohol Analyzer (Analox Instruments, Boston, MA), calibrated before and checked every 5-6 samples during each use, using a $200-\mathrm{mg} / \mathrm{dL}$ standard.

\section{Activated caspase-3 immunocytochemistry}

Additional PD 7 pups were treated with alcohol ( $n=3$, one-day treatment) or saline $(n=4)$ and used for immunocytochemical documentation of alcohol-induced activation of caspase-3 on PD 7, as previously reported by Olney et al. (2002). An antibody against the apoptosis marker, cleaved-caspase-3 (c-caspase 3; activated form, cleaved adjacent to Asp175; Cell Signaling Technology, Danvas, MA, USA) was used as published previously (Chen, Ozturk, Ni, Goodlett, \& Zhou, 2011). In our immunocytochemical procedure, one alcohol-treated and one control brain were pair-embedded together in a single gelatin block 
with careful rostrocaudal and dorsoventral alignments, and serial $40-\mu \mathrm{m}$ coronal sections were cut using a Leica VT 100S vibrating microtome. The 2-brain sections were then processed free-floating in the same vial and thus treated equally in all aspects of the immunocytochemical processing. Sections were incubated with $3 \% \mathrm{H}_{2} \mathrm{O}_{2}$ in $0.1 \mathrm{M}$ phosphate-buffered saline (PBS, pH 7.4) for 10 min and then washed in PBS and incubated in 1\% Triton X-100 in a phosphate buffer overnight. Sections were preincubated in PBS containing $0.1 \%$ Triton-X, $1.5 \%$ normal goat serum for $90 \mathrm{~min}$ before incubation with anticaspase-3 antibody (rabbit polyclonal, 1:150) overnight. The next day, sections were washed 3 times in PBS, and then incubated with a biotin-conjugated goat-anti-rabbit secondary antibody (Jackson ImmunoResearch Lab, 1:500) for 90 min. Sections were rinsed in PBS and then incubated in peroxidase-conjugated streptavidin (Jackson ImmunoReseach Lab, 1:500) for $60 \mathrm{~min}$. The peroxidase reaction was performed with $0.003 \% \mathrm{H}_{2} \mathrm{O}_{2}$ and $0.05 \%$ 3'3-diaminobenzidine (Sigma). The primary, secondary, and tertiary antibodies were diluted in PBS containing $0.1 \%$ Triton X-100 and $1.5 \%$ normal goat serum. All procedures were done at room temperature. All sections were Nissl-counterstained with methyl green to reveal background cells to identify brain regions. The staining of cleaved caspase- 3 for apoptosis has been validated and is comparable with Nile blue vital staining and TUNEL staining (Chen et al., 2011).

\section{Water maze apparatus and training}

Water maze training consisted of 4 trials per day for 10 consecutive days, in which a $9 \mathrm{~cm}$ (diameter) white platform was placed in a $125 \mathrm{~cm}$ (diameter) pool filled to within $25 \mathrm{~cm}$ of the rim of the tank with $26^{\circ} \mathrm{C}$ water made opaque by adding non-toxic white tempera paint (Dick Blick Art Materials, Galesburg, IL). The top of the submerged platform was $0.5 \mathrm{~cm}$ below the surface of the water. The testing room $(2.8 \times 2.9 \mathrm{~m})$ was illuminated by overhead fluorescent lights and contained fixed audio (radio kept at low volume) and visual reference cues (e.g., posters, bookshelf, sink, computer monitor, and experimenter). For groups given training to escape from a single platform location ("Place" training), the platform position remained constant throughout training; each Place squad was randomly assigned to 1 of 4 possible platform positions (in the center of 1 of the 4 quadrants). For the Random-training condition, a set of 4 random platform locations were designated for use on all training days (one per quadrant, offset from the center of the quadrant) but the sequence of escape locations across the 4 daily trials was varied randomly each day, such that the specific location of the platform on a given trial could not be predicted. The water was systematically stirred between mice to discourage the possibility of following scent trails left by previous animals. With this design, spatial learning can be inferred from the emerging difference in performance over training between the Place-trained groups, for which distal room cues can guide navigation to the platform location, and the Random-trained groups, for which the trial-to-trial location of the platform cannot be predicted based on room cues. Escape paths were monitored by a video camera interfaced with computer-controlled tracking software (HVS Image, Hampton, UK) and recorded by a JVC VCR.

The mice began training in the water maze at either PD 30 or PD 70 (Table 1), and the experimenter was blind to neonatal treatment condition. The computer-controlled tracking software recorded the moment-to-moment position of the animal in the pool for each trial, 
and obtained the following performance measures: escape latency, escape path length, swim speed, initial heading angle, and time spent near the perimeter of the pool (thigmotaxis).

Training trials consisted of placing the mouse in the pool (facing the rim of the tank) at 1 of 12 starting positions (corresponding to the 12 positions on a clock face, experimenter standing at 0600). The animal was allowed to swim for $60 \mathrm{~s}$ or until the platform was mounted. Different, pseudo-randomly determined starting points were used for each trial. Animals were not started within the same quadrant as the target, nor were start positions repeated on a given day. If a mouse was unable to find the platform, it was guided to the platform by the experimenter. All animals remained on the platform for $10 \mathrm{~s}$ before being returned to their respective incubators $\left(30^{\circ} \mathrm{C}\right)$ used to limit hypothermia during the intertrial interval. Mice were tested in squads of 3 or 4 , resulting in an intertrial interval of approximately $3 \mathrm{~min}$. At the end of each day of testing, the mice were allowed to remain in the incubators until warm and mostly dry (approximately $10 \mathrm{~min}$ ) before being returned to the vivarium.

On the 5th and 10th days of training, animals were subjected to a 60 -s probe trial, occurring approximately $5 \mathrm{~min}$ after the 4th training trial of the day. The platform was removed before the probe trial, and the animal's escape path was assessed via computerized video tracking. The program superimposed 4 virtual counting discs $(27 \mathrm{~cm}$, i.e., 3 times the size of the platform) over the pool in the location of each of the 4 possible platform positions used for the Place-training condition to calculate time spent in, latency to enter, and numbers of passes through each of the 4 virtual counting discs to quantify the spatial distribution of the search strategy of each animal. For Place-trained mice, the escape platform location used during training was designated as the target counter and compared to the average of the nontarget counter variables. For Random-trained mice, no platform was designated as the target, but all training positions were monitored separately to assess any place biases of the pool or testing room.

\section{Behavioral analyses}

Escape latencies and path lengths were highly correlated $(r=+0.91)$ and the patterns of outcomes on both measures of learning and performance were similar. Consequently, only path lengths will be reported, since this measure is less susceptible to non-specific performance effects such as floating in place or scrambling along the wall. To avoid litter effects, only one observation for each gender per treatment group per litter contributed to the analysis. The data were first analyzed using separate mixed analyses of variance (ANOVAs) within each neonatal treatment group, with training type (Place vs. Random) and gender as between-group factors and training day as a repeated measure. This analysis tested the $a$ priori hypothesis that acquisition of efficient escape performance (shorter distances as training progressed) should be significantly better in animals given Place training vs. those given Random training, and should yield significant main and/or interactive effects of training type. After determining the pattern of performance related to learning within each treatment group, the Place-training groups were analyzed separately with a mixed ANOVA with neonatal treatment group and gender as between-group factors and training day as a 
repeated factor. Specific post hoc comparisons between groups were performed using Fisher's protected least significant difference (LSD) $t$ tests $(a=0.05)$.

For the probe trials, the number of crossings through the platform regions and the time spent in each virtual target location were the primary measures of a learned search bias for a target location. For Place-trained animals, the trial time spent in the virtual target disc (in the target quadrant) and the average time spent in the 3 equivalent virtual non-target discs (in the 3 other quadrants) were analyzed using a mixed ANOVA with treatment group and gender as between-group factors and disc type (target vs. non-target) as a repeated measure. Specific within-subjects comparisons between target and non-target discs, as well as betweensubjects comparisons between treatment groups for the target discs, used protected LSD $t$ tests. For the Random-trained mice, the trial time spent in each of the 4 possible virtual disc locations was analyzed using a mixed ANOVA with treatment as a between-group factor and disc (training position) as a repeated factor.

\section{Results}

\section{Blood alcohol concentrations (BACs)}

The BACs on PD 7 are shown in Table 2. The mean $( \pm$ SEM) BAC on PD $7,1 \mathrm{~h}$ after the second alcohol injection, was $472( \pm 16) \mathrm{mg} / \mathrm{dL}$. The alcohol clearance rate was calculated to be $28.3 \mathrm{mg} / \mathrm{dL} / \mathrm{h}$.

\section{Caspase-3 immunocytochemistry}

As shown in Fig. 1, few cleaved-caspase-3 immunoreactive (im) cells were found in the control brains (left panels); in contrast, a large number of cleaved-caspase-3 im cells, most bearing neuronal morphology, were found in the alcohol-treated forebrains (right panels). This contrasting difference in cleaved-caspase- 3 im was found between all control and alcohol-treated pups evaluated. The cleaved-caspase- 3 im cells were distributed in cingulate cortex (Fig. 1A and B), striatum (Fig. 1C and D), and subiculum/distal CA1-CA2 of the hippocampal formation (Fig. 1E and F), and in the thalamus and parietal, temporal, and retrosplenial cortices (not shown). The pattern of caspase- 3 im cells in the cortices preferentially occurred in layer II and in layers IV and V of the frontal, cingulate, parietal, and temporal cortex, similar to that reported previously by Olney et al. (2002).

\section{Pre-weaning somatic growth}

Analyses of growth rates (Fig. 2) were performed separately for males and females using a 4 (neonatal treatment) $\times 9$ (day) repeated-measures ANOVA of body weights on PD 7 through PD 12 and on PD 15, 21, and 25. In cases where a litter had multiple mice of the same sex and treatment, scores were averaged such that only one score from each litter per treatment per sex were included in the analysis. Alcohol treatment significantly delayed weight gain in both sexes [treatment $\times$ day interaction, $F(24,680)=4.17, p<0.005$ for females; $F(24,712)$ $=3.62, p<0.005$ for males]. In females, both alcohol treatment groups weighed significantly less than the suckle controls from PD 7 to PD 25, but only the 3-day alcohol treatment group weighed significantly less than saline controls $(p<0.05)$. Alcohol treatment delayed body weight gain similarly in males from PD 7 to PD 25; however, both the 1- and 
3-day alcohol exposure groups weighed significantly less than both control groups. There were no significant treatment effects on adult body weights of male or female mice (obtained on P 63, 7 days prior to adult water maze training, Fig. 3).

\section{Adolescent water maze: acquisition path length}

An overall 4 (treatment) $\times 2$ (training) $\times 2($ sex $) \times 10$ (day) repeated-measures ANOVA confirmed the expected main effect of day $[F(9,10,535)=9.88, p<0.005]$, and mice given Place training showed significantly greater decreases in escape path lengths over training days relative to their Random-trained controls [training $\times$ day interaction: $F(9,1035)=5.21$, $p<0.005$; main effect of training: $F(1,115)=45.37, p<0.005$ ]. A significant main effect of neonatal treatment $[F(3,115)=6.67, p<0.005]$ was evident such that both neonatal alcoholtreatment groups had longer path lengths than their saline and suckle controls $(p<0.005)$.

To confirm the extent of place learning in each neonatal treatment condition, separate analyses were performed for each treatment comparing Place- and Random-trained groups, using a 2 (training) $\times 2($ sex $) \times 10$ (day) repeated-measures ANOVA. There were no significant main or interactive effects of sex on acquisition path length for any neonatal treatment, but females and males are presented in separate panels because sex differences did emerge on the probe trials (see below). Suckle-control mice (Fig. 4A and B) showed the expected divergence of path lengths over days between Place- and Random-trained groups, confirmed by main effects of training $[F(1,29)=25.49, p<0.005]$ and day $[F(9,261)=3.49$, $p<0.005$ ]. Saline-treated mice (Fig. 4C and D) show a similar divergence of Place and Random groups, confirmed by significant main effects of training $[F(1,27)=12.60, p<$ $0.005]$ and day $[F(9,243)=5.09, p<0.005]$. The PD 7 alcohol groups also showed divergence between place and random training (Fig. 4E and F), confirmed by a significant main effect of training $[F(1,31)=12.57, p<0.005]$ and day $[F(9,279)=3.43, p<0.005]$. The groups given the 3-day neonatal alcohol treatment (Fig. 4G and H) showed a modest separation between Place- and Random-trained groups that emerged only after the first 3 days of training [training $\times$ day interaction: $F(9,252)=2.29, p<0.05$ ].

To compare directly the neonatal treatment groups given Place training, a 4 (treatment) $\times 2$ $($ sex $) \times 10$ (day) repeated-measures ANOVA was performed on the data collapsed over sex (Fig. 5A). There was a significant main effect of treatment $[F(3,59)=6.83, p<0.005]$ in addition to the expected main effect of day $[F(9,531)=15.05, p<0.005]$. The longer path lengths of the two alcohol-treated groups were particularly evident over the last half of training (Fig. 5B), in which the PD 7 and the PD 7-9 alcohol-exposure groups had significantly longer escape path lengths over the last 5 days of training compared to both the suckle- and the saline-control groups, which did not differ from each other (Fisher's LSD post hoc tests, $p<0.05$ ). Nevertheless, the two alcohol-treated groups had significantly shorter path lengths than the Random groups, evidence that they did acquire some spatial learning. As place training progressed, the mice showed significantly less thigmotaxis time (swimming near the perimeter of the pool) [main effect of day: $F(9,531)=10.95, p<0.005$, data not shown], regardless of neonatal treatment or sex. A 4 (treatment) $\times 2($ sex $) \times 10$ (day) repeated-measures ANOVA revealed sex and treatment group differences in swimming speeds as training progressed [main effect of day: $F(9,531)=2.60, p<0.01$; 
alcohol treatment $\times$ day interaction: $F(27,531)=1.69, p<0.05$; sex $\times$ day interaction: $F(9,531)=4.01, p<0.005]$. Separate 4 (treatment) $\times 10$ (day) ANOVAs for each sex revealed the sex $\times$ day interaction was due to the increase in swimming speed over the course of training in female B6 mice [main effect of day: $F(9,270)=5.33, p<0.005$ ], whereas the swimming speeds of male B6 mice did not change. Once the analyses were separated by sex, the alcohol interactions were no longer significant for either male or female B6 mice.

Neonatal alcohol treatment did not affect swimming speeds of mice given Random training.

\section{Adolescent water maze: probe day 5}

A 4 (treatment) $\times 2($ sex $) \times 2$ (training) $\times 2$ (disc type: target vs. non-target) repeatedmeasures ANOVA on the probe trial indicated Place-trained mice spent more time in the target platform region of the pool [main effect of training: $F(1,118)=20.40, p<0.05$ ]. Repeated-measures ANOVA of the Place-trained groups yielded no main or interactive effects of neonatal treatment or sex (data not shown). Thus, as indicated by the amount of time spent in the virtual target disc, the search pattern at the end of training indicated that all 4 groups and both sexes had comparable biases toward the target location, though these target search biases were not as large as for the probe trial on day 10 (see below). There were no significant neonatal treatment effects on preference for the target location in either sex. There were no significant group or sex differences in overall path length, heading degree, or thigmotaxis time during the day 5 probe in the Place-trained groups. For the Random-trained mice (data not shown) a 4 (treatment) $\times 2$ (sex) $\times 4$ (disc location) repeatedmeasures ANOVA revealed no significant differences due to treatment, though there was a modest but significant bias toward disc locations in closest proximity to the experimenter [main effect of disc type: $F(3,180)=3.10, p<0.05$ ]. The neonatal alcohol treatment did not significantly affect overall path length or swimming speed on the probe trial, indicating the alcohol treatment did not significantly impair swimming performance.

\section{Adolescent water maze: probe day 10}

A 4 (treatment) $\times 2($ sex $) \times 2$ (disc type: target vs. non-target) repeated-measures ANOVA on the probe trial of the Place-trained groups confirmed that Place-trained mice spent significantly more time in the target discs than in the non-target discs [main effect of disc type: $F(1,49)=38.82, p<0.005$ ]. Although the overall ANOVA did not yield main or interactive effects of treatment, there was a significant sex $\times$ disc type interaction $[F(1,49)=$ $4.35, p<0.05]$, in which males showed a significantly stronger preference for the target platform disc than females, especially for the 2 control groups (compare Fig. 6A and B). Follow-up 4 (treatment) $\times 2$ (disc type) analyses of probe trials performed separately for females and males indicated that both males and females spent significantly more time in the target discs than in the non-target discs [main effect of disc type: $F(1,23)=9.45, p<0.005$ for females, Fig. 6A; $F(1,26)=32.51, p<0.005$ for males, Fig. 6B]. For the females, however, the saline controls were the only group to show a significant preference for the target platform region $(p<0.05)$. In contrast, for the males, the SC, SAL, and PD 7 groups all showed significant target disc biases ( $p<0.005$ for SC; $p<0.05$ for SAL and PD7 ALC), but the PD 7-9 ALC group failed to show a significant preference for the target platform 
region. There were no significant group or sex differences in overall heading angle, swimming speed, path length, or thigmotaxis time during the probe in the Place-trained groups.

For the Random-trained mice (Fig. 6C) a 4 (treatment) $\times 2(\operatorname{sex}) \times 4$ (disc location) repeated-measures ANOVA revealed no significant differences due to group or disc location in the time spent in the 4 virtual counting discs. The neonatal alcohol treatment did not impair swimming performance as indicated by overall path length and swimming speed on the probe trial. In fact, a significant treatment effect on the ANOVA of probe trial path lengths $[F(3,98)=3.20, p<0.05]$ was due to the significantly longer path lengths of the PD 7 alcohol group compared to the saline-control group and the PD 7-9 alcohol group ( $p<$ $0.05)$.

\section{Adult water maze: acquisition path length}

Mean daily path lengths during acquisition training for the adult groups are shown in Fig. 7 . An overall 4 (treatment) $\times 2$ (training) $\times 2($ sex $) \times 10$ (day) repeated measures ANOVA confirmed the expected overall decrease in escape path length over training days [main effect of day: $F(9,1044)=40.07, p<0.005]$, and groups given Place training decreased path lengths to a greater extent than their Random-trained counterparts [training $\times$ day interaction: $F(9,1044)=9.73, p<0.005$; main effect of training: $F(1,116)=40.40, p<$ 0.005]. The neonatal alcohol treatment significantly impaired acquisition [main effect of neonatal treatment: $F(3,116)=8.82, p<0.005]$, primarily due to the effect on the Placetrained groups [neonatal treatment $\times$ training interaction: $F(3,116)=5.55, p<0.005$ ]. There were no significant main or interactive effects of sex on adult acquisition in the overall ANOVA, but data in Fig. 7 are shown separately for males and females to facilitate comparison to adolescent data.

Each treatment group was analyzed separately using a 2 (training) $\times 2($ sex $) \times 10$ (day) repeated-measures ANOVA. For the suckle-control groups, the expected divergence in path lengths of the Place-trained mice relative to the respective Random-trained group was evident in both sexes (Fig. 7A and B), as confirmed by a significant training $\times$ day interaction $[F(9,252)=7.04, p<0.005]$ and main effects of training $[F(1,28)=71.23, p<$ $0.005]$ and day $[F(9,252)=18.38, p<0.005]$. Saline-treated groups had a similar divergence in path lengths of the Place and Random groups over days, confirmed by a significant training $\times$ day interaction $[F(9,279)=3.87, p<0.005]$, and significant main effects of training $[F(1,31)=42.21, p<0.005]$ and day $[F(9,279)=17.29, p<0.005$, Fig. $7 \mathrm{C}$ and $\mathrm{D}]$. Saline-treated males given Place training performed significantly better than their female counterparts, confirmed by a significant main effect of $\operatorname{sex}[F(1,31)=6.94, p<0.05]$ and sex $\times$ training interaction $[F(1,31)=5.63, p<0.05]$. For the PD 7 alcohol groups, the divergence between Place- and Random-training over days was not significant. The repeated-measures ANOVA yielded only a main effect of day $[F(9,261)=6.29, p<0.005]$, and there were no significant main or interactive effects of training or sex (Fig. 7E and F). Finally, for the PD 7-9 alcohol groups, divergence between Place- and Random-trained groups over days was limited, although the training $\times$ day interaction was significant $[F(9,252)=2.75, p<0.005]$, in addition to the significant main effect of day $[F(9,252)=$ 
5.35, $p<0.005]$; there were no main or interactive effects of sex. The remarkably limited divergence of the Place and Random groups in the adult PD 7 alcohol group stands in striking contrast to the clear training differences in the 2 control groups.

Comparison of the acquisition data for the 4 adult Place-trained groups (see Fig. 8A) clearly documents the spatial learning impairment of both the PD 7 and PD 7-9 alcohol groups. A 4 (treatment) $\times($ sex $) \times 10$ (day) repeated measures ANOVA of the Place-trained groups confirmed the effect of alcohol treatment [main effect of treatment: $F(3,56)=8.83, p<$ 0.005 ; treatment $\times$ day interaction: $F(27,504)=1.77, p<0.05$; main effect of day: $F(9,504)$ $=40.97, p<0.005]$. The acquisition deficits were especially apparent over the last half of training. A 1-way ANOVA and post hoc tests on mean path length averaged over the last 5 days of training (Fig. 8B) indicated that the PD 7 and the PD 7-9 groups both had average daily path lengths that were significantly longer than both control groups, and the 2 control groups did not differ from each other. The 2 alcohol-treatment groups did not differ from each other, nor did they differ from the combined Random-trained groups. Over the course of training, Place-trained mice spent significantly less time in thigmotaxis [navigating the perimeter of the pool; main effect of day: $F(9,504)=18.68, p<0.005]$. There was a significant effect of treatment group on time spent in thigmotaxis $[F(3,56)=3.54, p<0.05]$, and post hoc tests confirmed this effect was only significant in mice given alcohol on PD 79. Within the adult Random-trained groups, there was a significant effect of day $[F(9,540)=$ $7.74, p<0.005$ ], indicating that the groups did modestly reduce their escape latencies over the course of training, but path lengths did not differ by neonatal treatment. A 4 (treatment) $\times 2($ sex $) \times 10$ (day) repeated-measures ANOVA on swimming speed indicated that there were alcohol treatment effects on swimming speed [treatment $\times$ day interaction: $F(27,540)=$ $1.76, p<0.05]$. However, this interaction was driven by the slower swimming speeds of suckle controls $(p=0.052)$.

\section{Adult water maze: probe day 5}

Preference for the target platform-containing region during probe trials for the Place-trained groups (data not shown) were analyzed with a 4 (treatment) $\times 2$ (sex $) \times 2$ (disc type: target vs. non-target) repeated measures ANOVA. B6 mice given Place training spent more time in the target quadrant virtual disc than in the non-target virtual discs [main effect of platform type: $F(1,56)=18.08, p<0.005$ ]. Preference for the target disc location was significantly stronger in the control groups than in the alcohol-treated groups [main effect of treatment: $F(3,56)=3.48, p<0.05]$, treatment by platform type interaction $[F(3,56)=3.23, p<0.05]$, and there was a significant alcohol treatment $\times$ sex interaction $[F(3,56)=3.70, p<0.05]$. Separate analyses of each sex indicated that females given Place training expressed significant place biases to the target region [main effect of disc type: $F(1,31)=6.93, p<$ 0.05 ] that depended in part on treatment group [main effect of alcohol treatment: $F(3,31)=$ $5.92, p<0.05]$. Post hoc tests confirmed that the female suckle controls had significant search biases in favor of the target counter region $(p<0.005)$, whereas the differences between target and non-target search times in the saline-treated controls, PD 7, and PD 7-9 alcohol groups did not reach statistical significance. Place-trained males also significantly preferred the target location [main effect of disc type: $F(1,25)=10.25, p<0.05$ ], but this effect was not affected by neonatal alcohol treatment at this stage of training. A 1-way 
ANOVA on the time spent in the target discs confirmed significant group differences [main effect of treatment: $F(3,56)=3.56, p<0.05$ ], and the PD 7 and PD 7-9 groups spent significantly less time in the target counter than either control group (post hoc tests, $p<$ 0.05; data not shown). Looking at males and females separately, alcohol treatment significantly decreased the time spent in the target platform-containing disc in female B6 mice [main effect of alcohol treatment: $F(3,31)=3.35, p<0.05$ ], with mice given alcohol on PD 7-9 spending significantly less time in the target platform disc than suckle controls ( $p$ $<0.005)$. The alcohol treatment effect on time spent in the target platform disc among male mice failed to reach significance. There were no main or interactive effects of treatment or sex on path length, heading angle, swimming speed, or time near the edge of the pool (thigmotaxis) of Place-trained mice.

For the Random-trained groups, the time spent in the 4 virtual discs in the probe trial was relatively low ( $<8 \%$ of the total time) and was more evenly distributed than in the Placetrained groups. A 4 (treatment) $\times 2(\operatorname{sex}) \times 4$ (disc location) repeated-measures ANOVA revealed a main effect of disc $[F(3,165)=28.53, p<0.005]$ and a significant 4-way interaction $[F(9,165)=2.44, p<0.05]$. Post hoc comparisons indicated a small but significant preference for the discs nearest the location of the experimenter $(p<0.05)$. There were no main or interactive effects of treatment or sex on path length, swimming speed, or thigmotaxis time in the Random-trained mice.

\section{Adult water maze: probe day 10}

Probe trials for the Place-trained groups at the end of training were analyzed with a 4 (treatment) $\times 2($ sex $) \times 2$ (disc type) repeated-measures ANOVA. As seen in Fig. 9A, mice given Place training spent more time in the target quadrant virtual disc than in the non-target virtual discs [main effect of platform type: $F(1,56)=66.62, p<0.005$ ]. Preference for the target disc location was significantly stronger in the control groups than in the alcoholtreated groups [main effect of treatment: $F(3,56)=5.63, p<0.005$; treatment $\times$ platform type interaction: $[F(3,56)=4.30, p<0.01]$. Post hoc tests reveal that both alcohol-treatment groups were significantly impaired relative to both control groups $(p<0.01)$. To facilitate comparisons with the adolescent probe trial data, which revealed interactive effects of sex, data were analyzed separately for males and females. Control females showed the expected biases for the target platform disc $[F(1,6)=21.19, p<0.005$ for SC; $F(1,9)=12.33, p<$ 0.01 for SAL]. In contrast, neither alcohol-treatment group showed significant preferences for the target platform region. This same outcome held for males, such that both control groups significantly preferred the target platform disc, but neither alcohol-treatment group preferred the target platform disc $[F(1,7)=16.19, p<0.01$ for SC; $F(1,6)=65.06, p<0.005$ for SAL]. A 1-way ANOVA on the time spent in the target discs for each sex confirmed significant group differences among females [main effect of treatment: $F(3,31)=4.32, p<$ 0.05]; for males, there were no significant main or interactive effects of treatment. Post hoc tests in the females confirm that the PD 7-9 alcohol group spent significantly less time in the target platform disc than either the SC or SAL groups (see Fig. 8A). There were no main or interactive effects of treatment or sex on path length, heading angle, swimming speed, or thigmotaxis time for the Place-trained mice. 
For the Random-trained groups, the time spent in the 4 virtual discs in the probe trial was relatively low ( $<8 \%$ of the total time) and was more evenly distributed than in the Placetrained groups (see Fig. 8B). A 4 (treatment) $\times 2($ sex $) \times 4$ (disc location) repeated-measures ANOVA revealed only a main effect of disc $[F(3,177)=6.02, p<0.005]$, and post hoc comparisons indicated a small but significant preference for the discs nearest the location of the experimenter $(p<0.05)$. There were no main or interactive effects of treatment or sex on path length, heading degree, swimming speed, or thigmotaxis time for Random-trained mice.

\section{Discussion}

\section{Effects of PD 7 alcohol treatment}

The primary objectives of this study were to compare the effects of the heavy binge alcohol exposure on PD 7 in B6 mice on place learning performance in the Morris water maze in adolescence and adulthood, using the same model employed by Wozniak et al. (2004). We sought to further these findings to include a more extended 3-day binge exposure group. Based on the previous report by Wozniak et al. (2004), it was expected that the PD 7 treatment would produce massive spatial learning impairments when tested in adolescence, but the deficits would be greatly attenuated when tested in adulthood. This prediction was clearly disconfirmed, in that the adult mice given PD 7 alcohol treatment showed significant and obvious place learning impairments, even to the extent that there were no significant acquisition performance differences between the Place- and Random-trained groups. Moreover, although the PD 7 alcohol-treated group trained in adolescence showed significant impairments in place learning (relative to suckle and saline controls), they nevertheless showed significant learning relative to the Random-training control group. Furthermore, the Place-trained males given alcohol on PD 7 even showed a significant target location bias on the probe trial.

The current study was not designed to make direct comparisons between adolescent and adult animals, but it is clear that the spatial learning deficits produced by PD 7 alcohol treatment were not attenuated in adulthood. In contrast, Wozniak et al. (2004) found better place learning performance in adults (compared to the massive deficits they reported in adolescents), and suggested that the attenuated deficits could be due to recovery of function associated with emerging neuroplasticity. The results of the current study argue against such a recovery of function process and, to the contrary, indicate that the place learning differences become even more apparent as the mice mature beyond adolescence, as reported recently by Furumiya and Hashimoto (2011) using albino ddY mice.

\section{Place learning following PD 7-9 alcohol exposure}

The 3-day binge exposure on PD 7-9 caused the expected significant place learning impairment in both adolescence and adulthood, both in terms of acquisition path length measures and in the lack of an appropriate search bias in the probe trials. Given the comparable deficits in adulthood in the PD 7 and the PD 7-9 alcohol groups, the clear indication of these findings is that the neonatal binge alcohol treatments result in spatial 
learning deficits that persist into adulthood and, if anything, the effects are more apparent with maturation of the neural systems mediating place learning.

\section{Learning vs. performance considerations}

One important methodological aspect of the current study is that performance on distal cue (place) tasks was compared with performance on the same swimming task but for which the location of the submerged platform could not be predicted from trial to trial (random across 4 locations). In the original report by Morris et al. (1982), the Place-trained groups were similarly compared to groups given Random training similar to that used in this study. Comparison to Random training provides a means to confirm that the shorter distances and latencies acquired by the Place-trained groups as training progresses are due to spatial learning rather than other factors that may act to improve performance. This comparison gives confidence that the significant divergence in path lengths over days seen between the Place and Random groups for the suckle- and saline-control groups (both ages) and for the PD 7 alcohol group (adolescent) can be attributed to spatial learning and not just to general performance changes. Moreover, there were no significant neonatal treatment effects for either training age in performance in the Random-trained groups, either for the acquisition trials (path length, swimming speed) or for probe trials (total path length, swimming speed, time spent in the virtual counter discs). This lack of group differences supports the contention that the alcohol treatments did not alter the motivation to complete the task or the motor ability to perform the swimming task. Although fetal alcohol exposure has been shown to produce ocular abnormalities and impair vision (Strömland, 2004), studies in rats by Johnson and Goodlett (2002), and in mice by Wozniak et al. (2004) have shown that neonatal alcohol exposure does not impair an animal's ability to use local visual cues to find a visible platform. No studies, however, have directly evaluated alcohol treatment effects that may compromise visual acuity needed for distal cue identification, so the possibility remains that the alcohol treatments may alter visual abilities needed to discriminate locations of distal cues without affecting guidance to local visual cues.

Sex differences in neonatal alcohol-induced spatial learning deficits in adulthood have been reported in rats (Barron \& Riley, 1990; Blanchard, Hannigan, \& Riley, 1987; Johnson \& Goodlett, 2002; Kelly et al., 1988; O'Leary-Moore et al., 2006; Zimmerberg, Sukel, \& Stekler, 1991). Interestingly, these reports, with the exception of Kelly et al. (1988), point to a more severe neonatal alcohol-induced impairment in the spatial learning abilities of male rats. The current study found significant sex differences in the acquisition of place learning in saline-treated mice tested in adulthood and in probe trials of both adolescents and adults. More importantly, significant sex differences in the alcohol treatment effects in the water maze are apparent only in the probe trial on day 5 in adults, and this appears to be the first report in mice showing such a sex difference. Given the limited data on sex differences in the development of spatial learning in mice following neonatal alcohol exposure, this remains an important area for further research.

\section{Accounting for differences in outcomes}

Given the differences in outcomes of PD 7 binge alcohol exposure in this study compared to Wozniak et al. (2004), it is appropriate to consider what might account for those differences. 
Notably, in both studies, the spatial performance of the adolescent control groups (compared to adults) was relatively slow and average path lengths at asymptotic performance were longer. That is, the lack of effect seen in the adolescent mice in the current study may be associated with relatively less developed spatial learning by the control animals and slower acquisition over the 10 training days. However, the control animals in this study showed similar terminal path lengths to those of the Wozniak et al. (2004) study, so that alone cannot account for the differences in outcomes of the two studies. The blood alcohol concentrations of the current study were somewhat lower compared to the Wozniak et al. (2004) report ( $472 \pm 16 \mathrm{mg} / \mathrm{dL}$ compared to $510 \pm \sim 20 \mathrm{mg} / \mathrm{dL}$ ). This difference is likely not statistically significant and, more importantly, the BAC profile in the current study is well above that which reliably produces mass neurodegeneration, which we also demonstrated with the activated caspase-3 immunocytochemistry. It seems unlikely that the minor differences in measured peak BACs between the two studies can account for the major differences in outcomes.

There were a number of procedural differences between the two studies that may have contributed to the different outcomes. The pool used in this study was larger $(125 \mathrm{~cm}$ vs. $100 \mathrm{~cm}$ ), yielding $56 \%$ more searchable surface area, which can increase the sensitivity of the task (Carman \& Mactutus, 2001; Cronise, Marino, Tran, \& Kelly, 2001; Johnson \& Goodlett, 2002; Van Dam, Lenders, \& De Deyn, 2006; van der Staay, 2000) in ways that could increase the difficulty of the task, especially for the adolescent mice. In addition, the mice in the Wozniak et al. (2004) study were first given 2 consecutive days of cued training, in which the platform was visible. Initial training on the cued task is known to decrease the thigmotaxis tendencies and facilitate subsequent place learning, in part due to effects on general search strategies (Klatzky, 1988). Our choice to start acquisition with the spatial task (with no prior cued trials) was based on our interest in characterizing acquisition of flexible allocentric search strategies from onset of training. Although the mice in the current study did spend substantial time swimming near the tank wall (thigmotaxis) in the first day or two of training, that behavior decreased rapidly over the first few training days (as daily path length decreased); importantly, there were no differences in the thigmotaxis measure as a function of neonatal treatment. Other additional differences were that mice in the Wozniak et al. (2004) study were given longer periods of time on the platform after finding the submerged platform (30 s compared to $10 \mathrm{~s}$ in this study), were given two 4-trial sessions each day (compared to one), and were not kept in heated incubators between trials (as in this study). These procedural differences may have contributed to performance differences between the two studies (Hölscher, 1999; van der Staay, 2000), especially in the adolescent mice.

\section{Neuropathological correlates of place learning deficits in this mouse model}

The studies by Olney and colleagues using this mouse model have provided neuropathological evidence of significant apoptosis and degeneration of post-mitotic neuronal populations in specific layers of the cerebral cortex (frontal, cingulate, parietal, temporal, and retrosplenial cortex), hippocampal formation (CA1 and subiculum), striatum, anterior thalamus, and mammillary bodies (Olney et al., 2002; Wozniak et al., 2004). Many of these regions (e.g., hippocampal formation and entorhinal cortex; frontal, cingulate and 
parietal cortex) are known to be important components of the neural systems underlying spatial navigation, learning, and memory (Kolb, Sutherland, \& Whishaw, 1983; Morris et al., 1988, 1982; Olton \& Papas, 1979; Sutherland, Whishaw, \& Kolb, 1988; Whitlock, Sutherland, Witter, Moser, \& Moser, 2008; Woolley et al., 2013). Notably, in this PD 7 alcohol treatment in B6 mice and in other neonatal rodent binge models, the extent of apoptosis was relatively limited in the hippocampus (mainly in CA1/CA2), but was more robust in the subiculum, retrosplenial cortex, parietal cortex, and cingulate cortex (Ikonomidou et al., 2000; Olney et al., 2002; Wozniak et al., 2004). The cleaved-caspase-3 immunocytochemistry in the current study provided qualitatively similar patterns of neurodegeneration on PD 7 to those shown in Wozniak et al. (2004), suggesting that the alcohol-induced damage in our model was similar to that previously reported. The extent to which the PD 7-9 treatment may produce more apoptosis (cumulative over days) than the PD 7 treatment was not evaluated (and would be difficult to assess with immunocytochemistry, since the wave of cleaved-capapse- 3 expression from a daily binge episode dissipates within $24 \mathrm{~h}$. Nevertheless, it is notable that the PD 7 and the PD 7-9 groups did not differ significantly as adults in the extent of deficits in water maze acquisition. It is not yet clear what particular neural damage may account for the spatial learning deficits in this model, but given the rather limited damage in the hippocampus, it may relate more to damage in cortical, thalamic, or subicular regions that undergo substantial apoptosis, as suggested previously by Wozniak et al. (2004).

In summary, the findings of this study contrast with the previous results of Wozniak et al. (2004) and clearly indicate that binge-like alcohol treatment on PD 7 or PD 7-9 results in persistent deficits in spatial learning and memory. Although the differences in outcomes between the two studies remain unexplained, the effects on spatial learning are most likely due to the effects of alcohol on neural systems in the developing forebrain that mediate water maze learning. In light of the current findings, however, it is unlikely that there is the degree of recovery of function of spatial learning that was suggested by the earlier study, and that attenuation of spatial learning impairment is not likely to simply emerge as the animal matures from adolescence to adulthood.

\section{Acknowledgments}

This study was supported by NIH UO1 AA14829 to CRG and FCZ, part of Collaborative Initiative on Fetal Alcohol Spectrum Disorders (CIFASD) Consortium, and by NIH T32 AA007462 in support of JLW.

\section{References}

Barron S, Riley EP. Passive avoidance performance following neonatal alcohol exposure. Neurotoxicology and Teratology. 1990; 12:135-138. [PubMed: 2333065]

Blanchard BA, Hannigan JH, Riley EP. Amphetamine-induced activity after fetal alcohol exposure and undernutrition in rats. Neurotoxicology and Teratology. 1987; 9:113-119. [PubMed: 3657746]

Carman HM, Mactutus CF. Ontogeny of spatial navigation in rats: a role for response requirements? Behavioral Neuroscience. 2001; 115:870-879. [PubMed: 11508726]

Chen Y, Ozturk NC, Ni L, Goodlett C, Zhou FC. Strain differences in developmental vulnerability to alcohol exposure via embryo culture in mice. Alcoholism: Clinical and Experimental Research. 2011; 35:1293-1304. 
Clements KM, Girard TA, Ellard CG, Wainwright PE. Short-term memory impairment and reduced hippocampal c-Fos expression in an animal model of fetal alcohol syndrome. Alcoholism: Clinical and Experimental Research. 2005; 29:1049-1059.

Coleman LG Jr, Oguz I, Lee J, Styner M, Crews FT. Postnatal day 7 ethanol treatment causes persistent reductions in adult mouse brain volume and cortical neurons with sex specific effects on neurogenesis. Alcohol. 2012; 46:603-612. [PubMed: 22572057]

Cronise K, Marino MD, Tran TD, Kelly SJ. Critical periods for the effects of alcohol exposure on learning in rats. Behavioral Neuroscience. 2001; 115:138-145. [PubMed: 11256437]

Cudd TA. Animal model systems for the study of alcohol teratology. Experimental Biology and Medicine (Maywood). 2005; 230:389-393.

Dobbing J, Sands J. Comparative aspects of the brain growth spurt. Early Human Development. 1979; 3:79-83. [PubMed: 118862]

Dobbing J, Smart JL. Vulnerability of developing brain and behaviour. British Medical Bulletin. 1974; 30:164-168. [PubMed: 4467842]

Furumiya J, Hashimoto Y. Effects of ethanol exposure on spatial learning in mice during synaptogenesis. Nihon Arukoru Yakubutsu Igakkai zasshi = Japanese Journal of Alcohol Studies \& Drug Dependence. 2011; 46:250-259. [PubMed: 21702336]

Girard TA, Xing HC, Ward GR, Wainwright PE. Early postnatal ethanol exposure has long-term effects on the performance of male rats in a delayed matching-to-place task in the Morris water maze. Alcoholism: Clinical and Experimental Research. 2000; 24:300-306.

Goodlett CR, Kelly SJ, West JR. Early postnatal alcohol exposure that produces high blood alcohol levels impairs development of spatial navigation learning. Psychobiology. 1987; 15:64-74.

Hölscher C. Stress impairs performance in spatial water maze learning tasks. Behavioural Brain Research. 1999; 100:225-235. [PubMed: 10212070]

Ikonomidou C, Bittigau P, Ishimaru MJ, Wozniak DF, Koch C, Genz K, et al. Ethanol-induced apoptotic neurodegeneration and fetal alcohol syndrome. Science. 2000; 287:1056-1060. [PubMed: 10669420]

Ikonomidou C, Bosch F, Miksa M, Bittigau P, Vöckler J, Dikranian K, et al. Blockade of NMDA receptors and apoptotic neurodegeneration in the developing brain. Science. 1999; 283:70-74. [PubMed: 9872743]

Johnson TB, Goodlett CR. Selective and enduring deficits in spatial learning after limited neonatal binge alcohol exposure in male rats. Alcoholism: Clinical and Experimental Research. 2002; 26:83-93.

Kelly SJ, Goodlett CR, Hulsether SA, West JR. Impaired spatial navigation in adult female but not adult male rats exposed to alcohol during the brain growth spurt. Behavioural Brain Research. 1988; 27:247-257. [PubMed: 3358862]

Klatzky, RL. Allocentric and egocentric spatial representations: definitions, distinctions, and interconnections. In: Freksa, C.; Habel, C.; Wender, KF., editors. Spatial cognition - An interdisciplinary approach to representation and processing of spatial knowledge. Berlin: SpringerVerlag; 1988. p. 1-18.

Kodituwakku PW. Defining the behavioral phenotype in children with fetal alcohol spectrum disorders: a review. Neuroscience and Biobehavioral Reviews. 2007; 31:192-201. [PubMed: 16930704]

Kolb B, Sutherland RJ, Whishaw IQ. A comparison of the contributions of the frontal and parietal association cortex to spatial localization in rats. Behavioral Neuroscience. 1983; 97:13-27. [PubMed: 6838719]

Light KE, Belcher SM, Pierce DR. Time course and manner of Purkinje neuron death following a single ethanol exposure on postnatal day 4 in the developing rat. Neuroscience. 2002; 114:327337. [PubMed: 12204202]

Livy DJ, Miller EK, Maier SE, West JR. Fetal alcohol exposure and temporal vulnerability: effects of binge-like alcohol exposure on the developing rat hippocampus. Neurotoxicology and Teratology. 2003; 25:447-458. [PubMed: 12798962]

Morris RGM. Spatial localization does not require the presence of local cues. Learning and Motivation. 1981; 12:239-260. 
Morris RG, Downes JJ, Sahakian BJ, Evenden JL, Heald A, Robbins TW. Planning and spatial working memory in Parkinson's disease. Journal of Neurology, Neurosurgery, and Psychiatry. 1988; 51:757-766.

Morris RG, Garrud P, Rawlins JN, O'Keefe J. Place navigation impaired in rats with hippocampal lesions. Nature. 1982; 297:681-683. [PubMed: 7088155]

O’Leary-Moore SK, McMechan AP, Mathison SN, Berman RF, Hannigan JH. Reversal learning after prenatal or early postnatal alcohol exposure in juvenile and adult rats. Alcohol. 2006; 38:99-110. [PubMed: 16839856]

Olney JW, Tenkova T, Dikranian K, Muglia LJ, Jermakowicz WJ, D’Sa C, et al. Ethanol-induced caspase-3 activation in the in vivo developing mouse brain. Neurobiology of Disease. 2002; 9:205-219. [PubMed: 11895372]

Olton DS, Papas BC. Spatial memory and hippocampal function. Neuropsychologia. 1979; 17:669682. [PubMed: 522981]

Pauli J, Wilce P, Bedi KS. Spatial learning ability of rats following acute exposure to alcohol during early postnatal life. Physiology \& Behavior. 1995; 58:1013-1020. [PubMed: 8577871]

Rice D, Barone S Jr. Critical periods of vulnerability for the developing nervous system: evidence from humans and animal models. Environmental Health Perspective. 2000; 108(Suppl 3):511-533.

Riley EP, McGee CL. Fetal alcohol spectrum disorders: an overview with emphasis on changes in brain and behavior. Experimental Biology and Medicine (Maywood). 2005; 230:357-365.

Strömland K. Visual impairment and ocular abnormalities in children with fetal alcohol syndrome. Addiction Biology. 2004; 9:153-157. discussion 159-160. [PubMed: 15223541]

Sutherland RJ, Whishaw IQ, Kolb B. Contributions of cingulate cortex to two forms of spatial learning and memory. The Journal of Neuroscience. 1988; 8:1863-1872. [PubMed: 3385478]

Tomlinson D, Wilce P, Bedi KS. Spatial learning ability of rats following differing levels of exposure to alcohol during early postnatal life. Physiology \& Behavior. 1998; 63:205-211. [PubMed: 9423960]

Tran TD, Kelly SJ. Critical periods for ethanol-induced cell loss in the hippocampal formation. Neurotoxicology and Teratology. 2003; 25:519-528. [PubMed: 12972065]

Van Dam D, Lenders G, De Deyn PP. Effect of Morris water maze diameter on visual-spatial learning in different mouse strains. Neurobiology of Learning and Memory. 2006; 85:164-172. [PubMed: 16290194]

van der Staay FJ. Effects of the size of the morris water tank on spatial discrimination learning in the CFW1 mouse. Physiology \& Behavior. 2000; 68:599-602. [PubMed: 10713304]

West JR, Goodlett CR. Teratogenic effects of alcohol on brain development. Annals of Medicine. 1990; 22:319-325. [PubMed: 2291839]

Whitlock JR, Sutherland RJ, Witter MP, Moser MB, Moser EI. Navigating from hippocampus to parietal cortex. Proceedings of the National Academy of Sciences of the United States of America. 2008; 105:14755-14762. [PubMed: 18812502]

Woolley DG, Laeremans A, Gantois I, Mantini D, Vermaercke B, Op de Beeck HP, et al. Homologous involvement of striatum and prefrontal cortex in rodent and human water maze learning. Proceedings of the National Academy of Sciences of the United States of America. 2013; 110:3131-3136. [PubMed: 23382228]

Wozniak DF, Hartman RE, Boyle MP, Vogt SK, Brooks AR, Tenkova T, et al. Apoptotic neurodegeneration induced by ethanol in neonatal mice is associated with profound learning/ memory deficits in juveniles followed by progressive functional recovery in adults. Neurobiology of Disease. 2004; 17:403-414. [PubMed: 15571976]

Zimmerberg B, Sukel HL, Stekler JD. Spatial learning of adult rats with fetal alcohol exposure: deficits are sex-dependent. Behavioural Brain Research. 1991; 42:49-56. [PubMed: 2029344] 

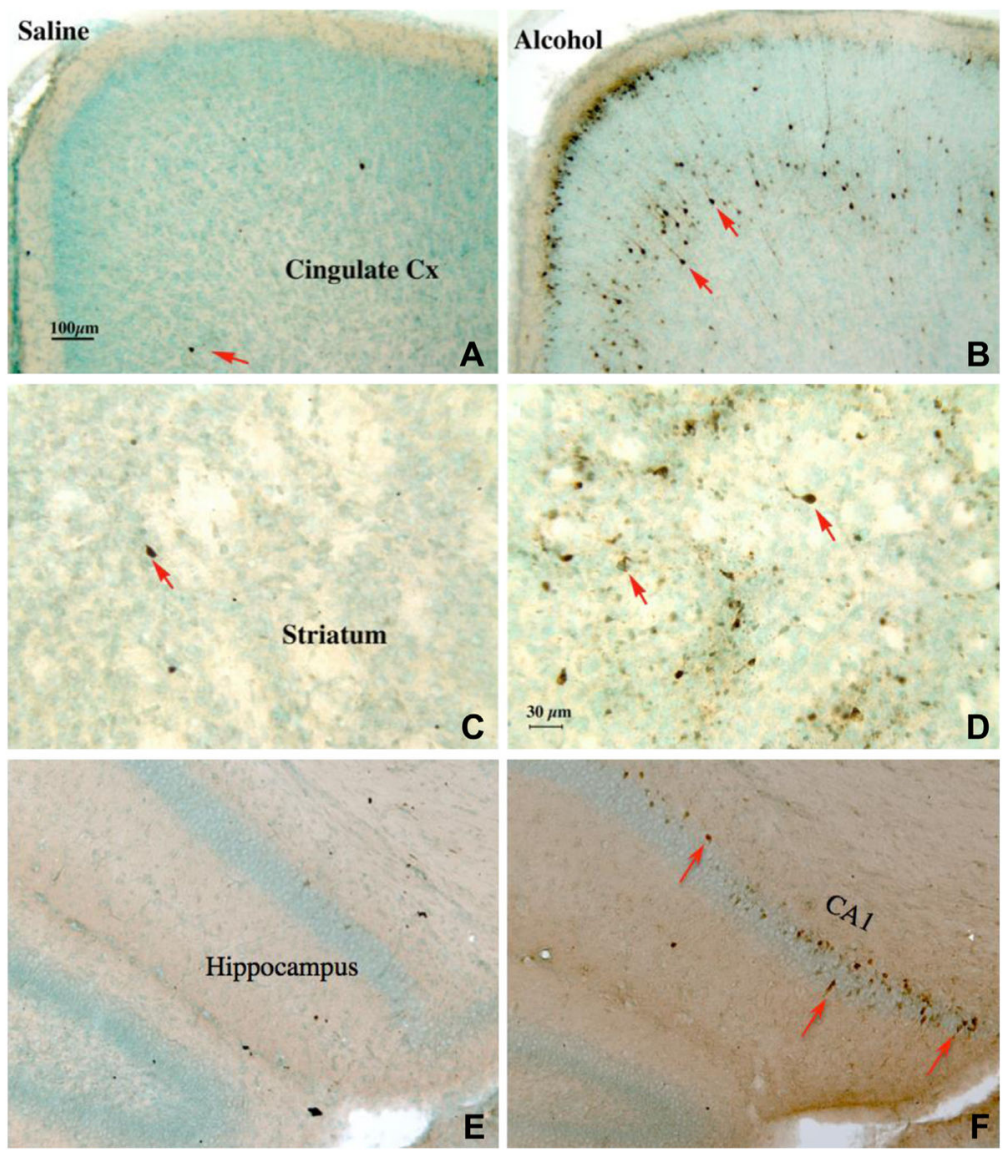

Fig. 1.

Alcohol-induced apoptosis indicated by immunocytochemistry of cleaved-caspase-3, shown in photomicrographs of salinetreated (left panels) and alcohol-treated (right panels) mice for cingulate cortex (top panels), striatum (middle panels), and hippocampal formation (bottom panels). Alcohol was administered to B6 mice on PD 7 ( $2.5 \mathrm{~g} / \mathrm{kg}$ twice a day, $2 \mathrm{~h}$ apart, s.c.) and the pups were perfused for immunocytochemistry $6 \mathrm{~h}$ after the second alcohol injection. In saline-treated mice, very few cleaved-caspase-3 positive neurons were seen (A, C, E; arrows). In contrast, many caspase-3-positive neurons were evident in the cingulate cortex (B) [as well as parietal, temporal and retrosplenial cortices (not shown)], striatum (D), and hippocampal formation CA1/2 [as well as subiculum (not shown)] (F) of mice treated with alcohol. Scale bars: A, B, E, F = $100 \mu \mathrm{m} ; \mathrm{C}, \mathrm{D}=$ $30 \mu \mathrm{m}$. 


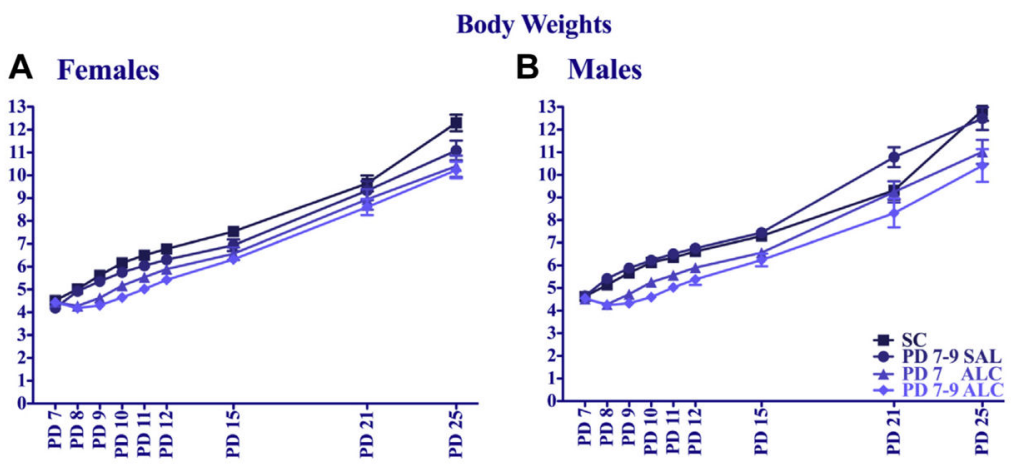

Fig. 2.

Body weights (group means \pm SEM) during and after neonatal treatment for female (A) and male (B) mice. 


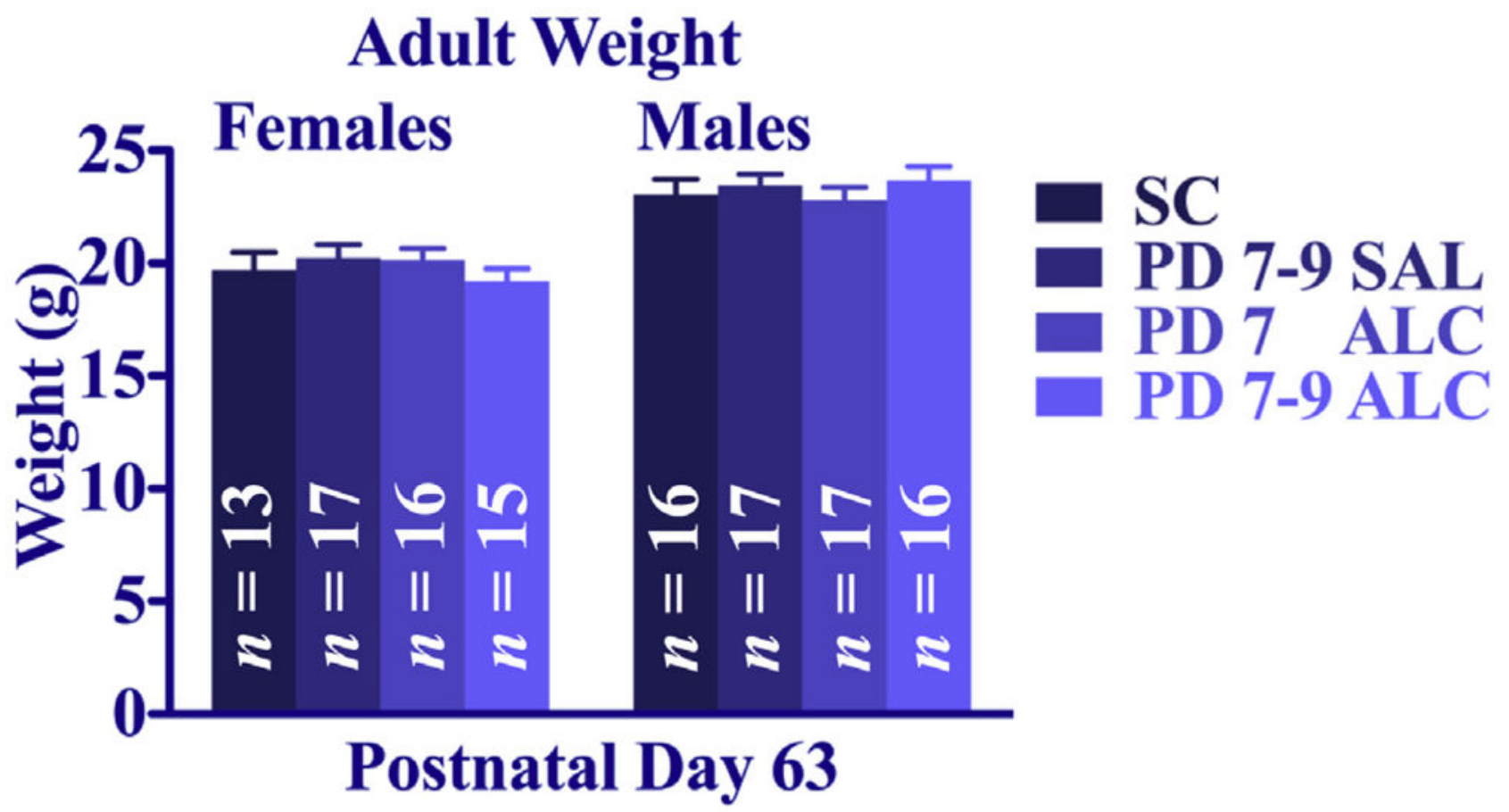

Fig. 3.

Adult body weights (group mean \pm SEM) on P 63 . There were no significant group differences. 


\section{Adolescent Acquisition x Sex}
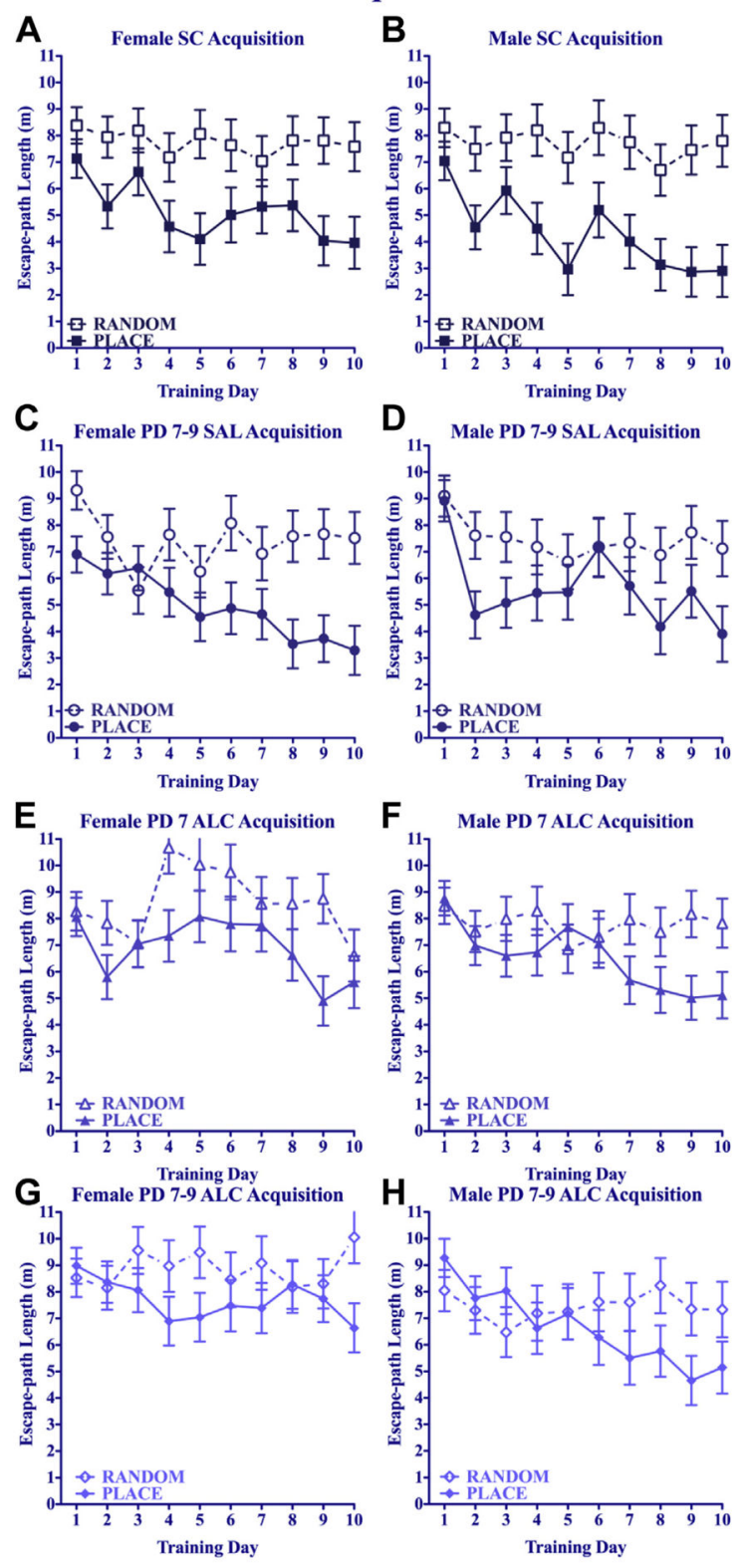

Fig. 4.

Adolescent water maze acquisition. Mean daily escape path length $( \pm$ SEM) for Place- and Random-trained groups. Broken lines represent animals given Random training and solid lines represent Place-trained animals. 


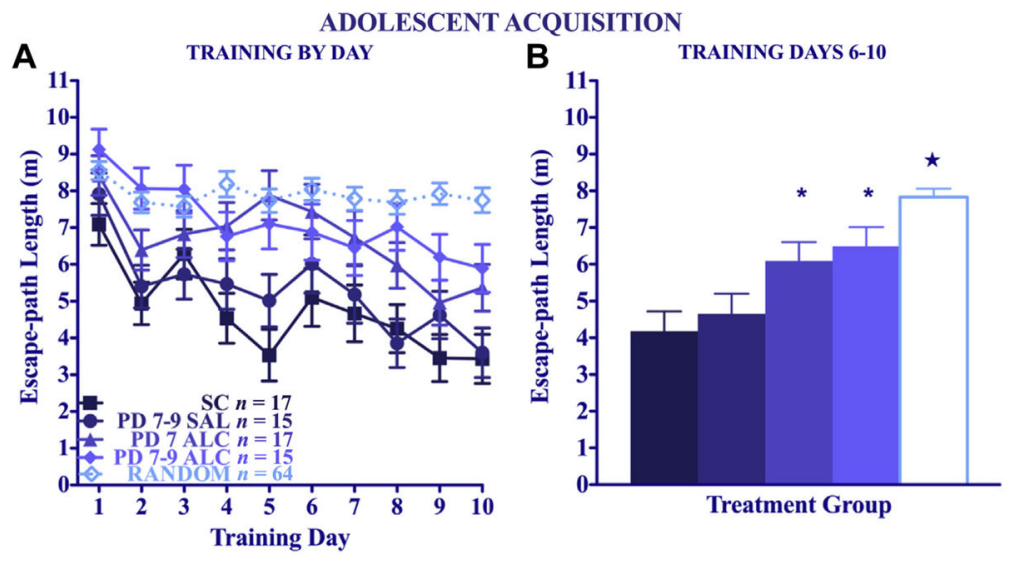

Fig. 5.

Adolescent water maze acquisition. Mean daily escape path lengths ( \pm SEM) for Place-trained animals (A) over days and (B) collapsed across the last 5 training days (*Significantly different from both control groups, $p<0.05)\left({ }^{\star}\right.$ Significantly different from all groups, $p<0.05)$. 

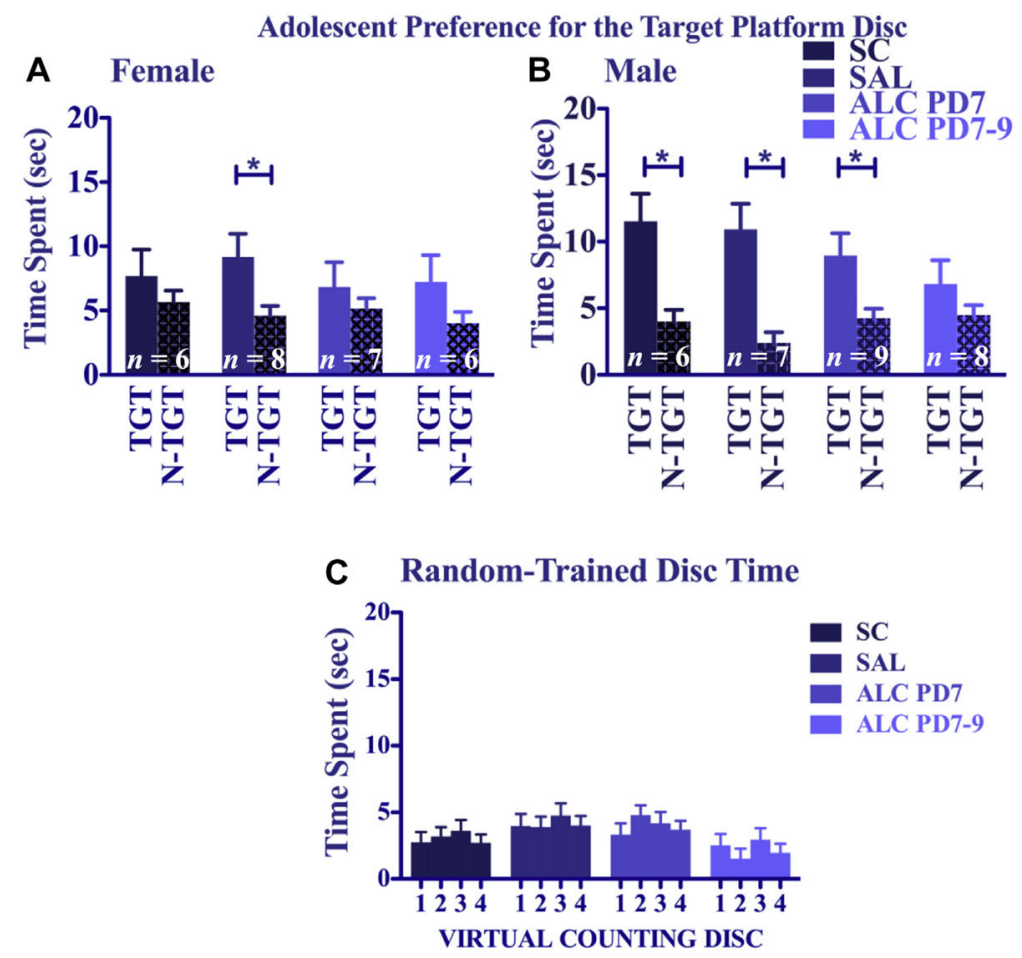

Fig. 6.

Adolescent water maze probe trial (day 10). Time spent in the virtual counting discs in the 4 platform locations by adolescent animals [Panel A: Females; Panel B: Males]. Time spent in the virtual counting disc of the target platform vs. the average time spent exploring the 3 other platform discs of the target platform vs. the average time spent exploring the 3 other platform counting discs. TGT - target counter discs; N-TGT - average of non-target counter discs. [Panel C] Time spent in each of the 4 place virtual counting discs by Random-trained animals. *Represents significant differences between the target and non-target platform disc within the treatment group. 
Adult Acquisition x Sex
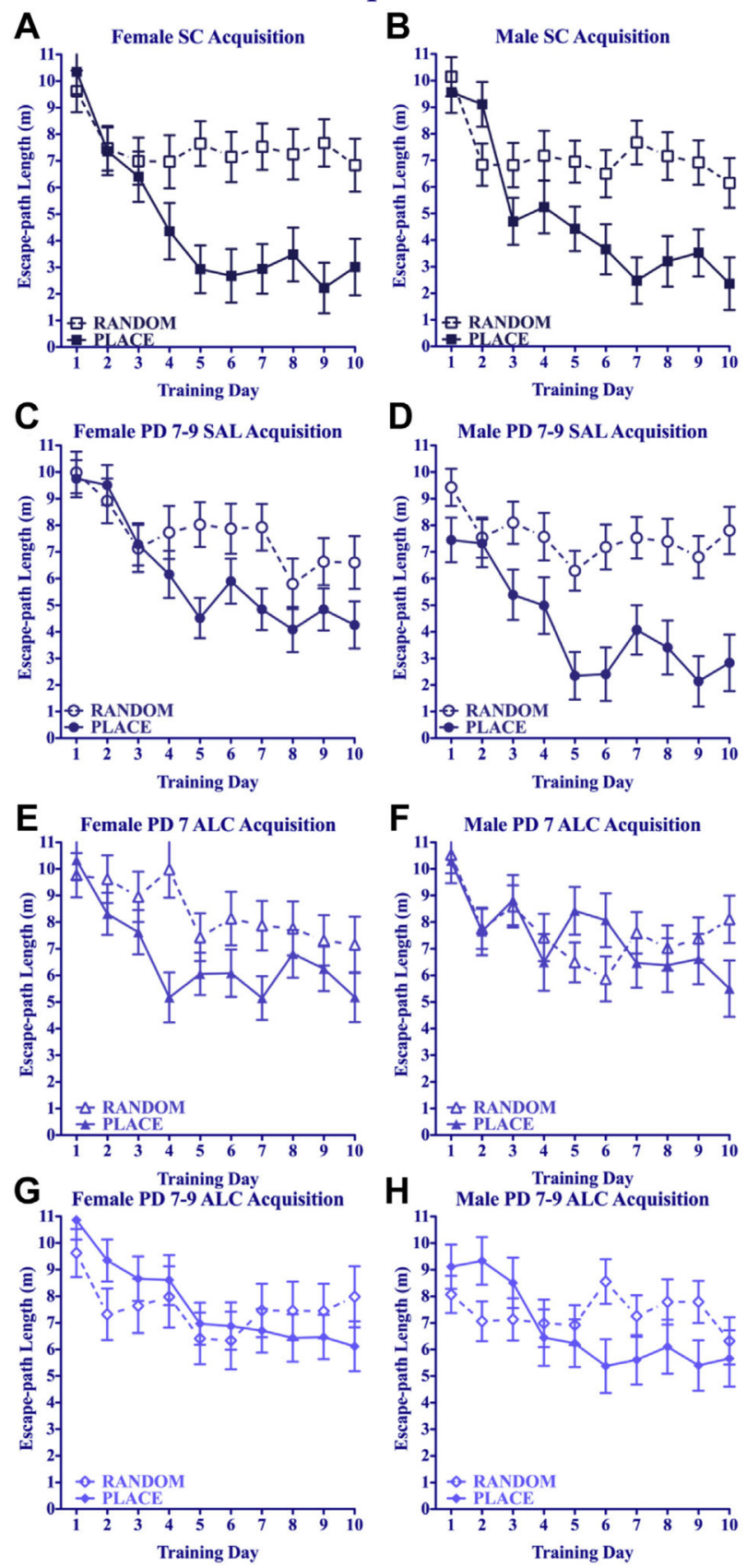

Fig. 7.

Adult water maze acquisition. Mean daily escape path length $( \pm$ SEM) for Place-and Random-trained groups. Broken lines represent Random groups. Solid lines represent Place groups. 


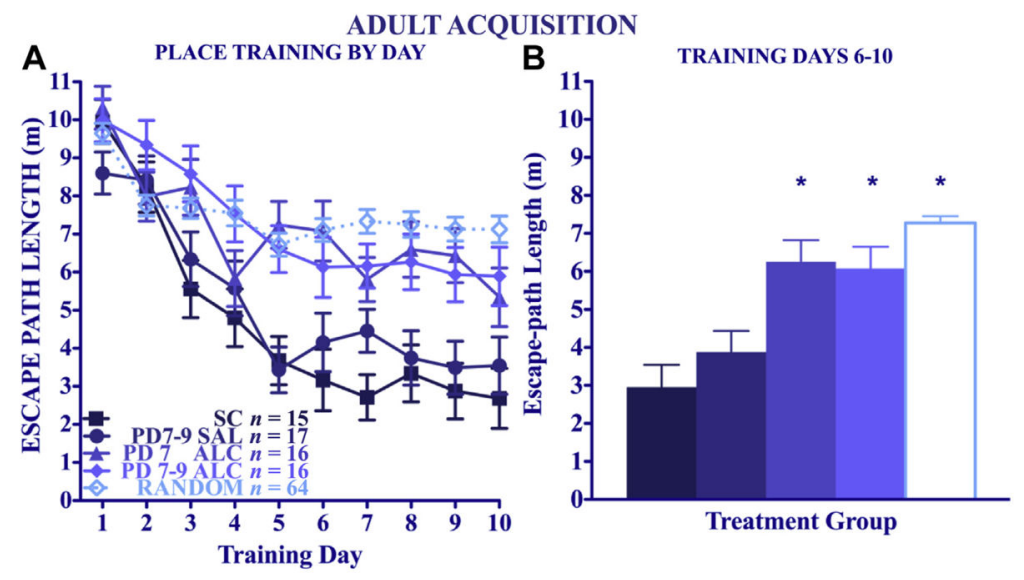

Fig. 8.

Adult water maze Acquisition. Mean daily escape path lengths ( \pm SEM) for Place-trained adult animals (A) over days and (B) collapsed across the last 5 training days. *Significantly different from both control groups, $p<0.05$. 


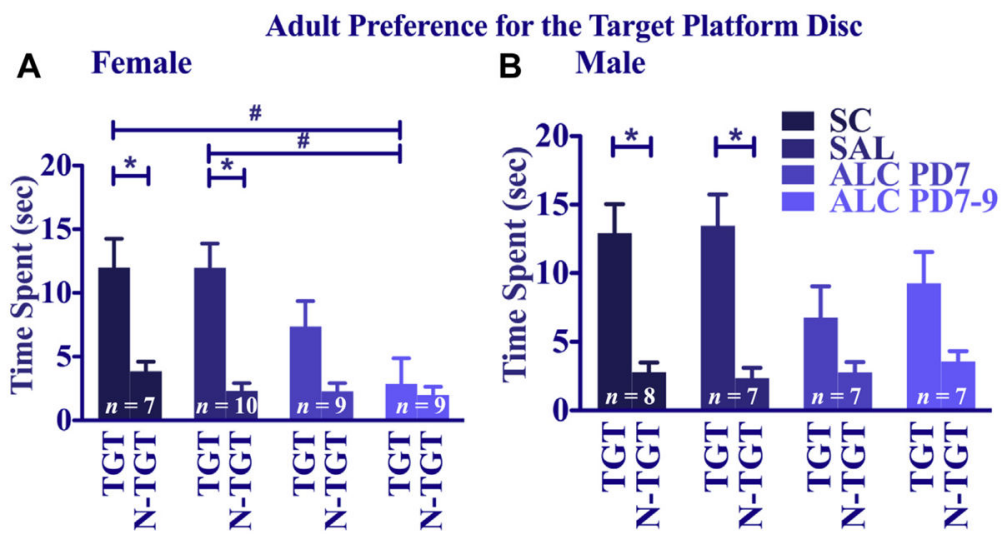

C Random-Trained Time in All Dises

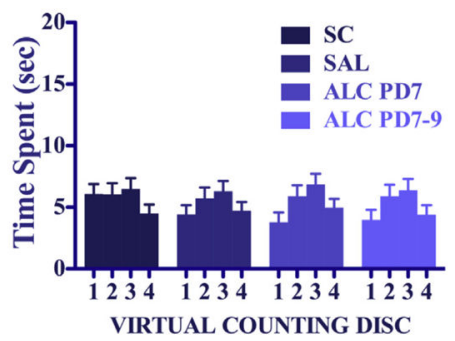

Fig. 9.

Adult water maze probe. Time spent in virtual counting disc in the 4 platform positions [Panel A: Females; Panel B: Males]. Time spent in the virtual counting disc of the target vs. average time spent in the other 3 non-target virtual counting discs $(* p<$ $0.05, \# p<0.01$ ). [Panel C] Time spent in each of the 4 virtual counting discs by adult Random-trained animals. *Represents significant differences between the target and non-target platform disc within the treatment group. "Represents significant differences between the amount of time spent in just the target platform disc between groups. 


\section{Table 2}

Mean ( \pm SEM) blood alcohol concentrations (in $\mathrm{mg} / \mathrm{dL}$ ) on PD 7.

\begin{tabular}{lll}
\hline $\mathbf{1} \mathbf{h}$ & $\mathbf{4 h}$ & $\mathbf{7} \mathbf{h}$ \\
\hline $472 \pm 16$ & $385 \pm 8$ & $302 \pm 13$ \\
$n=13$ & $n=12$ & $n=11$ \\
\hline
\end{tabular}

Trunk blood samples were obtained 1,4 , and $7 \mathrm{~h}$ after the second alcohol injection on PD 7 . 